\title{
Imaging of Healthy and Malaria-Mimicked Red Blood Cells in Polydimethylsiloxane Microchannels for Determination of Cells Deformability and Flow Velocity
}

\author{
Liliana Vilas Boas ${ }^{1,2}$, Rui Lima ${ }^{3,4}$, Graça Minas ${ }^{1}$, \\ Carla S. Fernandes ${ }^{2}$, and Susana O. Catarino ${ }^{1(\bowtie)}$ \\ ${ }^{1}$ Microelectromechanical Systems Research Unit (CMEMS-UMinho), \\ University of Minho, Guimarães, Portugal \\ scatarino@dei.uminho.pt \\ 2 ESTiG, IPB, C. Sta. Apolónia, Bragança, Portugal \\ ${ }^{3}$ MEtRiCS, University of Minho, Guimarães, Portugal \\ ${ }^{4}$ CEFT, University of Porto, Porto, Portugal
}

\begin{abstract}
Imaging analysis techniques have been extensively used to obtain crucial information on blood phenomena in the microcirculation. In the present study, it is intended to mimic the effects of malaria on the red blood cells (RBCs), by changing their properties using a different concentration of glutaraldehyde solution. The effects of the disease in stiffing RBCs were evaluated using polydimethylsiloxane microchannels that comprise contractions with $10 \mu \mathrm{m}$ width and measuring the cells deformability and the flow velocity in healthy and modified conditions. The obtained results show a decrease in the RBCs deformability and in the flow velocity with the presence of glutaraldehyde, when compared to the behavior of healthy RBCs samples. Therefore, it can be concluded that, using image analysis (ImageJ \& PIVLab), it is possible to measure the deformability of the RBCs and the flow velocity and, consequently, obtaining a correlation between the difference of velocities/deformabilities in the microchannels. In the future, this correlation can be used to relate the RBCs behavior with the various stages of malaria. This study can be a starting point for establishing the development of new malaria diagnostic systems towards point-of-care lab-on-a-chip devices.
\end{abstract}

\section{Introduction}

More than 3 billion of people are at risk of contracting malaria, which is one of the most severe parasitic diseases and causes between 1-2 million deaths per year [1]. The disease is predominantly widespread in regions that lack proper infrastructure and living conditions, worsening the chances of infection for the population. Additionally, the disease has very generic symptoms, which make it difficult to diagnose. Early stage detection is crucial since it greatly increases the possibility of an effective treatment, increasing the survival rate and reducing the transmission of the disease. Currently, the most used diagnostic methods in endemic regions are microscopy [2] and rapid 
diagnostic tests by immunochromatographic assays [3]. These techniques, despite being widely used, have limitations regarding the sensitivity, specificity, required time to perform the assays, cost and the need for specialized technicians and/or laboratories. Therefore, microfluidic systems can be an alternative to the current diagnostic methods $[4,5]$, since they offer advantages in terms of sample preparation and analysis (low volume of samples, easy handling, low cost and fast processing), eliminating the need for specialized personnel [4]. Taking into account the existent microtechnologies, it will be possible to integrate into a single chip, besides the microfluidic channels, sensors, actuators and microelectronics, enhancing the possibility of creating a fully automated and portable diagnostic device for malaria [5].

The malaria infection in the human red blood cells (RBCs) goes through several stages as the infection spreads. The parasite causes biochemical, optical and morphological changes in the RBCs, making these cells more rigid and thicker, which consequently causes an increase in the blood viscosity [6]. One of the most important blood properties for an early diagnosis of this disease is the deformability of the RBCs, since it is directly related to the changes that the parasite causes throughout the evolution of malaria. There have been reported in the literature several methods for measuring the deformability of RBCs in order to detect the presence of malaria. Examples of these methods are filtration [7], ektacytometry [8], optical tweezers [9], micropipette aspiration [10] and microchannels constrictions [11]. In order to develop and evaluate these methods, it is essential to synthetically mimic the malaria behavior in RBCs, for testing the efficiency and reproducibility of the methods, without the constant need for infected samples, improving the laboratorial safety of the tests. Thus, this work proposes the use of glutaraldehyde for mimicking the malaria effect in the RBCs. The cells will be rigidified and their behavior, relatively to deformability and flow velocity, will be compared to healthy RBCs. The evaluation of the RBCs deformability and flow velocity will be performed in a microchannel with an abrupt constriction, followed by an abrupt expansion. This approach (previously studied by the research team [12]) takes advantage of the potential of these geometries to deform cells due to shear and extensional flows generated by the sudden constrictions. The cells behavior will be captured by a high speed camera and the obtained images will be processed in two software tools (ImageJ and PIVLab) for determining both the RBCs deformability and the flow velocity.

\section{Materials and Methods}

This section presents the details of the microchannels fabrication, experimental setup and samples used to perform the experimental tests.

\section{- Microchannels Fabrication}

For the accomplishment of the experimental work, a microfluidic device with polydimethylsiloxane (PDMS) microchannels was microfabricated using a soft lithography technique, using SU-8 molds $[13,14]$. The microchannels have a $25 \mu \mathrm{m}$ height in order to reduce the flow volume and make it easier to observe the RBCs flow. Additionally, 

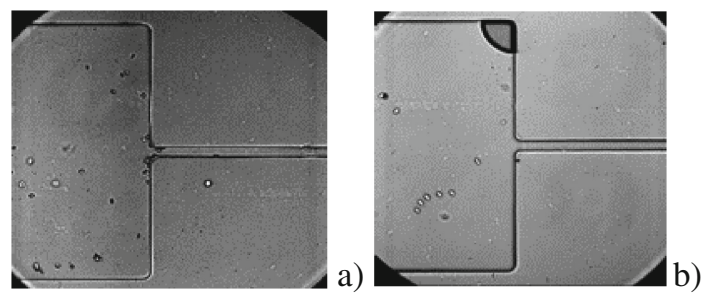

Fig. 1. Detail of the $10 \mu \mathrm{m}$ width contraction of the PDMS microchannel (a) entrance; (b) outlet. Magnification: 40x.

the microchannels have an abrupt contraction (at a $90^{\circ}$ angle) with a $10 \mu \mathrm{m}$ width and $780 \mu \mathrm{m}$ length (seen in Fig. 1), designed to force the cells to deform when crossing it.

- Samples

Samples containing human RBCs (hematocrit $=0.5 \%)$ in Dextran40 (Dx40) were used. Human RBCs have typical diameters in the $6 \mu \mathrm{m}-8 \mu \mathrm{m}$ range [15]. The Dx40 solution (used as a plasma-volume expander to prevent the RBCs sedimentation) was synthetically produced, by mixing $8 \mu \mathrm{L}$ of $\mathrm{CaCl} 2$ with $201 \mu \mathrm{L}$ of $\mathrm{KC}, 7.35 \mathrm{~mL}$ of $\mathrm{NaCl}$ and $5 \mathrm{~g}$ of $\mathrm{Dx} 40$ (for $1 \mathrm{M}$ solution). These samples were then modified with $0.00625 \%, 0.0125 \%, 0.025 \%$ and $0.08 \%$ glutaraldehyde solutions to mimic the malaria effect by rigidifying the cells at different levels. Blood components separation was performed by centrifuging the blood samples for $15 \mathrm{~min}$ at $2000 \mathrm{rpm}$, at room temperature. After that, RBCs were re-suspended and washed twice in a physiological solution (PSS) with a concentration of $0.9 \%$. For the glutaraldehyde exposed samples, cells were incubated for 10 min, washed again in PSS, re-suspended in Dex40 and used right away.

- Experimental setup

For the microfluidic experiments, the device containing the microchannel was placed on the stage of an inverted microscope (IX71; Olympus). A flow rate of $0.1 \mu \mathrm{L} / \mathrm{min}$ was controlled using a syringe pump (KD Scientific). The images of the flowing RBCs were captured using a high speed camera (Phantom v7.1; Vision Research) at 2000 frames/s rate and exported to a computer to be analyzed.

\section{Image Analysis}

The images imported from the high speed camera to the computer were treated and analyzed using two software tools: ImageJ and PIVLab [16, 17].

- ImageJ

ImageJ was used to perform a pre-treatment to the acquired frames and to determine the RBCs deformability. Initially, the desired image sequence was imported and the crop function was executed to define the region of interest (rectangle with $308 \times 332 \mu \mathrm{m}$ ), as shown in Fig. 2. 




Fig. 2. Example of a cut-off of the transfer zone $(308 \times 332 \mu \mathrm{m})$, using the crop function (the arrows indicate the flow direction).

Then, with the Z-Project function, the selected frames were stacked in order to obtain an average of the frames. Following this step, the sequence of images under analysis were subtracted by the averaged frame, eliminating all static objects. With that processing step, the resultant frames contained only the visible RBCs, without any additional information. Finally, using a threshold function, the images were converted into binary images.

The ImageJ software was also used to measure the cell size in order to calculate the deformability index (DI) of the RBCs. Therefore, using the region of interest (ROI) Manager function, it was possible to follow the RBCs and calculate their deformation rate along the microchannel, with and without glutaraldehyde, as shown in Fig. 3.
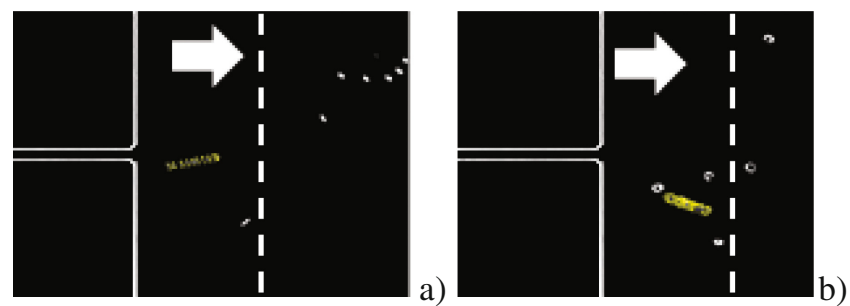

Fig. 3. Follow-up of RBCs in the outlet of the microchannel contraction. (a) deformed/stretched healthy cells with $0 \%$ glutaraldehyde; (b) chemically modified cells with $0.08 \%$ glutaraldehyde in the original non-deformed shape (the arrows indicate the flow direction; the dashed lines represent the expansion area after the outlet).

\section{- PivLab}

In order to determine the average velocity values of the RBCs, the images were analyzed using the PIVLab image analysis software, integrated in MATLAB.

Initially, the pre-treated images were imported into the software and calibrated (relatively to their dimensions and time between frames). Then, the particle motion was analyzed between all the frames and the instantaneous velocities were calculated by the variation of the distance traveled by the RBCs between each time step.

Then, the average velocity vectors were calculated in the $x$ and $y$ directions and the velocity field of each sample was determined, followed by the application of a filter to 
smooth the images and remove the high frequencies, which could indicate spikes of velocity without physical significance.

Then, the $x$ and $y$ components of the average velocity for each analyzed pixel were exported to Microsoft Excel, and the overall flow vectors were calculated based on the equation:

$$
\left|U_{x y}\right|=\sqrt{U_{x}^{2}+U_{y}^{2}}
$$

As it is possible to verify in Fig. 4, the velocity of the RCBs is significantly higher in the zone of the narrowing entrance, reasoning that the abrupt transition causes the increase of the velocity of the RBCs.

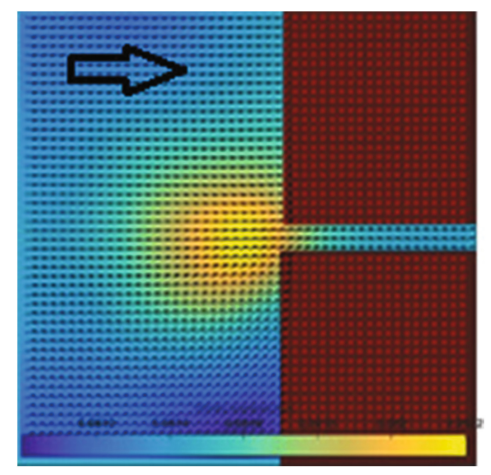

Fig. 4. Velocity distribution of the healthy RBCs (with $0 \%$ glutaraldehyde) in the microchannel with a narrowing width of $10 \mu \mathrm{m}$ (the arrow indicates the flow direction).

\section{Results and Discussion}

This section presents some preliminary results of the comparison between the healthy and chemically modified RBCs. As observed in the previous section (Sect. 3), it is possible to detect in Fig. 3a difference between the deformation of the healthy RBCs (Fig. 3(a)), which are deformed/stretched, and the deformation of glutaraldehyde modified RBCs (Fig. 3(b)), which not deform and keep their original circular shape. Figure 5 presents the DI for healthy and glutaraldehyde modified RBCs, at the entrance and exit of the microchannel contraction, as well as at the expansion area after the contraction (in Fig. 3, this expansion area is located right after the dashed line, and corresponds to the region where the RBCs recover their original shape). The results agree with the ones presented in Fig. 3. As the percentage of glutaraldehyde increases, the cells become more rigid, as shown in Fig. 5. While the healthy cells deformed at the entrance of the contraction and recovered to the initial form after reaching the microchannel expansion area, the modified RBCs did not deform and had difficulty to cross the contraction. 


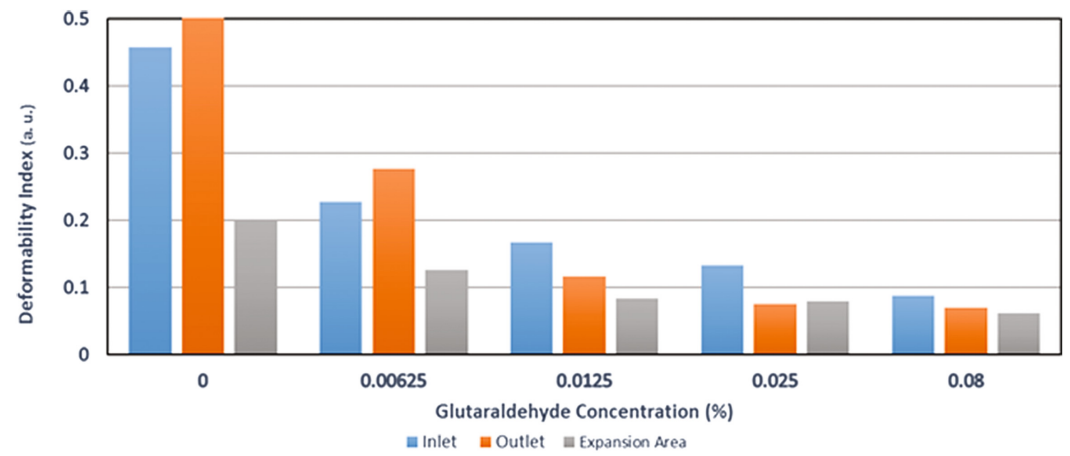

Fig. 5. DI for healthy and glutaraldehyde modified RBCs, at the entrance (blue column), exit (orange column) and expansion area (gray column) of the microchannel contraction.

Figure 6 displays an example of the velocity for healthy and glutaraldehyde modified RBCs, at the entrance of the microchannel contraction (blue column) and at the expansion area after the contraction exit (gray column). When the authors evaluated the average pixel velocity in the high velocity regions, it was found that the malaria-mimicked RBCs (glutaraldehyde modified cells) presented lower velocities than healthy RBCs, leading to the conclusion that the increase of the RBCs rigidity causes an increase of the viscosity and, consequently, a decrease of the flow velocity, as observed in Fig. 6.

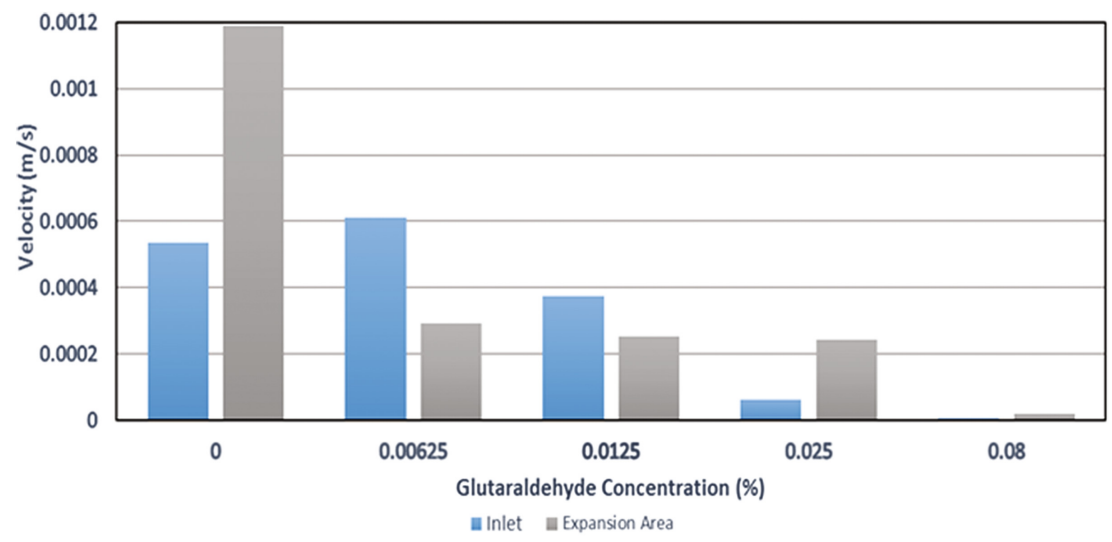

Fig. 6. Velocity for healthy and glutaraldehyde modified RBCs, at the entrance of the micro-channel contraction (blue column) and at the expansion area after the contraction exit (gray column). 


\section{Conclusions and Future Work}

This work presented an approach for measuring the deformability and flow velocity of healthy and chemically modified RBCs, attempting to mimic the effect of malaria in the RBCs and establish a relation between flow velocity and RBCs deformability. It can be concluded that the increase in the RBC stiffness causes the decrease of the RBCs deformability and cell shape recovering capacity and causes the decrease of the cells velocity.

It can also be concluded that there is a relationship between the deformability and the velocity of the cells. The higher the deformability of the RBCs, the greater the flow velocity. However, future work must be performed with different glutaraldehyde concentrations, in order to obtain a complete calibration curve that represents the deformability/velocity relation.

Therefore, despite being still a challenge, this work will be a valuable contribution for the development of simple, reagent-free, inexpensive and accurate new malaria diagnostic systems towards point-of-care lab-on-a-chip devices.

Acknowledgments. This work was supported by FCT with the reference project UID/EEA/04436/ 2013, by FEDER funds through the COMPETE 2020 - Programa Operacional Competitividade e Internacionalização (POCI) with the reference project POCI-01- 0145-FEDER- 006941. S.O. Catarino thanks the FCT for the SFRH/BPD/108889/2015 grant, supported by national funds from Ministérios da Ciência, Tecnologia e Ensino Superior and by FSE through the POCH - Programa Operacional Capital Humano. The authors thank Diana Pinho from the IP Bragança for providing the blood samples and for the support in the experimental tests.

\section{References}

1. World Health Organization: World Malaria Report 2008. World Health Organization (2008)

2. Wongsrichanalai, C., Barcus, M.J., Muth, S., Sutamihardja, A., Wernsdorfer, W.H.: A review of malaria diagnostic tools: microscopy and rapid diagnostic test (RDT). Am. J. Trop. Med. Hyg. 77, 119-127 (2007)

3. Murray, C.K., Gasser Jr., R.A., Magill, A.J., Miller, R.S.: Update on rapid diagnostic testing for malaria. Clin. Microbiol. Rev. 21, 97-110 (2008)

4. Hou, H., Bhagat, A., Chong, A., Mao, P., Tan, K., Han, J., Lim, C.: Deformability based cell margination - a simple microfluidic design for malaria-infected erythrocyte separation. Roy. Soc. Chem. 10, 2605-2613 (2010)

5. Catarino, S., Silva, L., Mendes, P., Miranda, J., Lanceros-Mendez, S., Minas, G.: Piezoelectric actuators for acoustic mixing in microfluidic devices-numerical prediction and experimental validation of heat and mass transport. Sens. Actuators B Chem. 205, 206-214 (2014)

6. Maichomo, M.W., McDermott, J.J., Arimi, S.M., Gathura, P.B., Mugambi, T.J., Muriuki, S. M.: Study of brucellosis in a pastoral community and evaluation of the usefulness of clinical signs and symptoms in differentiating it from other flu-like diseases. Afr. J. Health Sci. 7, 114-119 (2000)

7. How, T., Black, R., Hughes, P.: Hemodynamics of vascular prostheses. Adv. Hemodynamics Hemorheology 1, 373-423 (1996) 
8. Tomaiuolo, G.: Biomechanical properties of red blood cells in health and disease towards microfluidics. Biomicrofluidics 8, 051501 (2014)

9. Lim, C., Dao, M., Suresh, S., Sow, C., Chew, K.: Large deformation of living cells using laser traps. Acta Mater. 52, 1837-1845 (2004)

10. Hochmuth, R.: Micropipette aspiration of living cells. J. Biomech. 33, 15-22 (2000)

11. Zhao, R., Antaki, J.F., Naik, T., Bachman, T.N., Kameneva, M.V., Wu, Z.J.: Microscopic investigation of erythrocyte deformation dynamics. Biorheology 43, 747-765 (2006)

12. Paço, D., Lima, R., Minas, G., Catarino, S.O.: Red blood cells deformability as a malaria biomarker. In: Proceedings of the IEEE 5th Portuguese Meeting on Bioengineering (ENBENG), pp. 16-18 (2017)

13. Faustino, V., Catarino, S.O., Lima, R., Minas, G.: Biomedical microfluidic devices by using low-cost fabrication techniques: a review. J. Biomech. 49, 2280-2292 (2016)

14. Pinto, V.C., Sousa, P.J., Cardoso, V.F., Minas, G.: Optimized SU-8 processing for low-cost microstructures fabrication without cleanroom facilities. Micromachines 5, 738-755 (2014)

15. Rossetto, J., Abatti, P.: Análise da Passagem da Célula Vermelha do Sangue Através de Poros Cilíndricos. Trends Appl. Comput. Math. 3, 193-202 (2002). (in Portuguese)

16. ImageJ - Image processing and analysis in java. https://imagej.nih.gov/ij/

17. Thielicke, W., Stamhuis, E.J.: PIVlab - Time-Resolved Digital Particle Image Velocimetry Tool for MATLAB. https://figshare.com/articles/PIVlab_version_1_35/1092508 